OPergamon Press plc

\title{
MECHANICS OF TRANSPORT PHENOMENA IN MULTI-COMPONENT SESSILE DROPS WITH SOLIDIFICATION*
}

\author{
Yeong-Jen $\mathrm{Su}^{* *}$, Wen-Jei Yang and Jiaching Liu \\ Department of Mechanical Engineering and Applied Mechanics \\ The University of Michigan \\ Ann Arbor, Michigan 48109-2125
}

\section{ABSTRACT}

(Communicated by J.P. Hartnett and W.J. Minkowycz)

\begin{abstract}
An experimental study is performed to determine the mechanics of transport phenomena in multicomponent sessile drops with internal solidification. These drops are cooled at the center of the drop base or over the enter base of the drop. Both the interfacial and internal flow structures are examined by means of laser shadowgraphy, while the microstructures are investigated using a microscope-video system. Three different degrees of cooling rate are imposed on the drops: low, medium and high cooling. It is disclosed that the center cooling at a low rate produces a radial flow induced by component separation but without solidification and Marangoni type (surface-tension induced) flow. In contrast, a high cooling rate results in Marangoni convection accompanied by solidification but no component separation. The effect of Prandtl number on the Marangoni flow velocity is determined. The interfacial disturbance is suppressed by center cooling but not by bulk cooling which induces the formation of Bernard cells. The impact of flow patterns on the mechanical property of melt solidification is discussed.
\end{abstract}

\section{Intreduction}

The performance reliability of modern communication and computer electronics depends strongly upon the quality of the semi-conductor single crystals. One of the most important problems in the growth of semi-conductor single crystals is the homogeneity of the electrical resistivity. It has been disclosed that fluctuations of the electrical resistivity in such a crystal are caused by inhomogeneous dopant distribution which is due to the timewise variations in the growth rate. One factor leading to the unsteady crystal growth is unsteady convection in the liquid domain during the course of crystal growth.

\footnotetext{
" The work reponed was partially supported by the NASA Lewis Research Center under the grant number NAG3903.

** Presently with the Institute of Nuclear Energy Research, P. O. Box 3-3, Lung-Tan, Taiwan 32500, R.O.C.
} 
In many other material processes involving solidification, including a variety of casting and welding processes, extraction, purification, pharmacy as well as polymer production, the quality of the products is significantly affected by the thermal-fluid transport process during solidification.

A number of important physical phenomena have been identified to take place during solidification. In pure substances, the flow in liquid domain is induced by temperature gradients, the motion of the solid-liquid interface, or the expansion or contraction during solidification. In addition, the surface temperature gradient causes a surface tension gradient to generate a flow know as thermocapillary convection. These flows together with local heat transfer near the solid/liquid interface, determine the solidification rate and interfacial structure.

The phase-change of binary mixtures differs in many aspects from solidification of pure substances. Besides the convective phenomena mentioned above, the double-diffusive effects, the interdendritric fluid flow within the mushy zone, and the diffusocapillary convection caused by surface solutal gradients contribute significantly to the bulk flow in the liquid domain. In most systems, chemical components have different solubilities in the solid and liquid phases. Hence, during phase-change, a chemical species may be preferentially incorporated or rejected at the solid/liquid interface. This phenomenon complicates solid front geometry and interfacial instability. The central issue of convection in melts and crystal growth has been treated in a large number of publications [for example, 1-4]. In addition, several reviews on the subject have appeared during the past decade [for example, 5-8]. The reviews indicate that most of the attention has been focused on the influence of buoyancy and double-diffusive transport on flow and instability phenomena. Less effort has been devoted to investigate the effects of surface tension on solidification or crystal growth.

In a small-scale flow system or in a low-gravity environment, surface tension plays a dominant role in natural convection. There are two basic modes of flow induced by surface tension gradients: One is called Marangoni instability, with the gradients normal to the free surface. The other is named Marangoni convection, with the gradients along the free surface. The two modes are referred to as Marangoni effects [5]. Those induced by thermal and concentration gradients are called thermocapillary and diffusocapillary effects, respectively.

The Marangoni effects on interfacial instability of pure and binary liquid systems were studied by Castillo and Velarde $[9,10]$. Natural convection in sessile drops is of interest, since the liquid volume is not bounded by container walls. The effects of surface tension on natural convection in both pure and binary liquid drops evaporating on a plate were systematically investigated in references 11 through 14 . It was revealed that the morphology of sessile drops exhibited stable, substable, and unstable types as viewed by laser shadowgraphy. Stable type is characterized by an image with a perfect circular periphery, unstable type by a saw-toothed periphery, and subtable 
type by one between the two limits [11]. Two important dimensionless parameters governing the interfacial instability of binary sessile drops were found to be the excessive free energy of surface and the Crispation number $[13,14]$. An interfacial flow map was constructed which defined the domains of stable and unstable regions. The morphology of evaporating pure liquid sessile drops changed when point cooling was applied at the base center $[15,16]$.

Evaporation induces a temperature gradient, resulting in the generation of surface tension force on the drop surface. A doughnut-shaped, thermocapillary-induced vortex region was found to exist around the growing solid phase inside an evaporating drop [1517]. Flow visualization studies were performed on binary-component, sessile drops [18] and sessile drops with internal solidification [19].

In the present study, a shawdowgraph-schlieren system and a microscope-video system are employed to study the mechanics of transport phenomena in multi-component, sessile drops with solidification. The information obtained from the study will be useful to the enhancement of solid or crystal quality under reduced-gravity environment where both thermo-and diffuso-capillary effects on solidification and crystal growth are dominant.

\section{Experimental Apparatus and Procedure}

Figure 1 depicts a schematic of a shadowgraph-schlieren system. It consisted of a $35 \mathrm{MW}$ Helium-Neon laser, a pin hole, a test plate, two aluminized mirrors, a lens, a mask plate, a screen, and a camera. The mask plate was a $1 \mathrm{~mm}$ thick glass plate marked with a $0.5 \mathrm{~mm}$ diameter dark spot which was positioned at the focal point of the lens. Thus, it constituted the traditional schlieren technique with the knife edge being replaced by the dark spot. The solid lines in Fig. 1 illustrated the passage of laser beams which were completely blocked by the dark spot instead of being partially cut off by the knife edge as in the convectional schlieren method. The dashlines depicted the passage being deflected by the drop's lens effect, which was eventually projected on the screen for display and photography. The image was recorded by a Polaroid camera using the type 57, ASA 3000 films.

The local, internal flow behavior and the pattern of interface between the separated components were observed by means of a microscope-vide system. The system, as shown in Fig. 2, consisted of a Leitz model Metallux 3 microscope, an electrostatic focusing vidicon TV camera, a 700 line horizontal resolution black and white video monitor, and a multifunction VCR. Extrabrilliant No. 53 aluminum powder (Bronz Powder, Inc.) was used as tracer to follow the liquid motion.

The test plate was a $75 \times 25 \times 1 \mathrm{~mm}$ glass plate. A drop of multi-component liquid was carefully placed on the test plate by means of a micro syringe. Two cooling methods were employed to impose a temperature field: 




FIG 1

A schematic diagram of shadowgraph-schlieren system

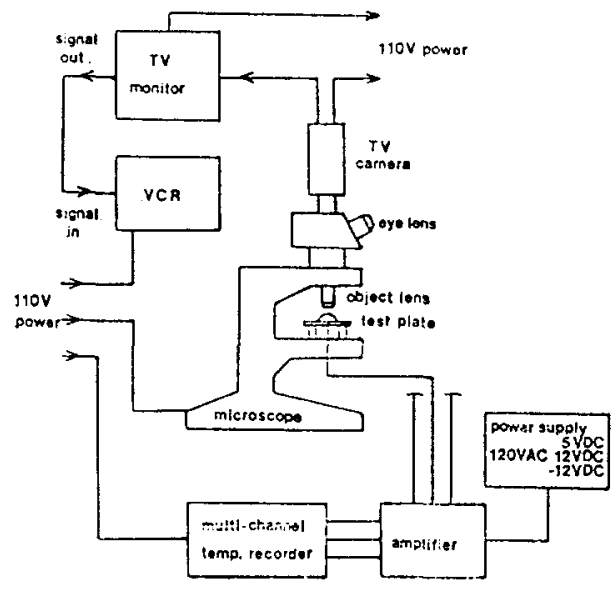

FIG 2

A schematic diagram of microscope-video system

The center-cooling method was to cool at the base center of a drop. A hole, $0.813 \mathrm{~mm}$ in diameter, was drilled on the test plate. A silver wire of equal diameter was inserted through the hole with its tip flush with the upper surface of the test plate. Cyanoacrylate was used to seal the very small gap. In performing the experiment, the loose end of the conducting wire was inserted in between two small pieces of dry ice, which were encased in a Styrofoam enclosure. Then, the test plate was put in the designated position of the shadowgraph or microscope-video system. A drop was carefully placed on the test plate with its base center located at the wire end.

The bulk-cooling method was devised to cool the entire base of the drop. The glass plate without a hole or conducting wire was surrounded by several pieces of dry ice. A drop was then placed on the evenly precooled test plate. When an appropriate degree of cooling was achieved, separation and surface tension-induced flow occurred in multi-component drops.

\section{Results and Discussion}

Several binary and trinary-component liquid drops of different compositions were investigated. Some typical results of dimethyl sulfoxide/oleic acid, tetrachloride/methylene chloride,and dimethyl sulfoxide/oleic acid/p xylene are presented here. The components of the first binary liquid drop, dimethyl sulfoxide and oleic acid, are characterized by a very slow evaporation rate with melting points near room temperature. In contrast, the components of the second binary liquid system, tetrachloride and methylene chloride, have a very fast evaporation rate and a rather low melting temperature. The physical properties of these pure liquids are presented in Table 1. 
Two methods of cooling are imposed on sessile drops which lead to internal solidification. One is the center cooling method with three distinct intensities of cooling: low, medium and high rates. Another is the bulk cooling method. The study is directed to determine the mechanics of two flow problems: interfacial instability and internal convection. The former is exhibited in the form of surface morphology and can be explored by means of laser shadowgraphy. The global view of the internal convection accompanied by solidification is obtained by the use of laser shadowgraphy, while the local phenomena is revealed using the microscope-video system.

\section{Interfacial Instability (i.e. surface morphology)}

\section{1-a. No cooling case}

Figure 3 is the laser shadowgraph of a $50 \%$ (by volume) dimethyl sulfoxide/50\% oleic acid drop without cooling. (a), (b) and (c) correspond to the initial (immediately after the placement of a drop), intermediate (after 5 minutes) and final (after 20 minutes) stages of evaporation, respectively. As pure liquids, both dimethyl sulfoxide and oleic acid drops were characterized by unstable interfacial structures throughout the entire evaporation process $[15,16]$. An examination of Fig. 3 indicates that the mixing has a temporary effect on the morphology of the binary drop during the initial and intermediate stages of evaporation process. At the final stage of evaporation, the true characteristics of the interfacial structure will appear.
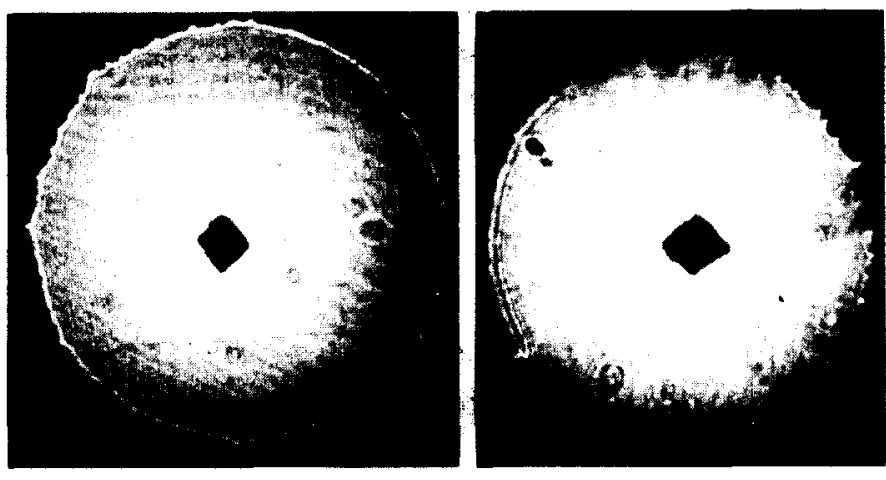

(a) (b)

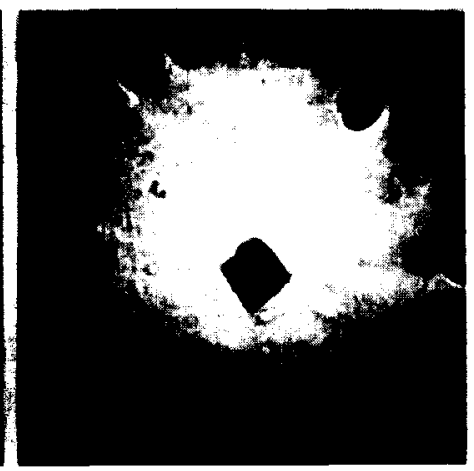

(c)

FIG 3

A laser shadowgraph of a $50 \%$ dimethyl sulfoxide-50\% oleic acid drop without cooling, (a) initial, (b) intermediate, and (c) final stages 
The interfacial instability of a sessible drop which is exhibited in the form of surface morphology is governed by two dimensionless parameters. Since a drop is so tiny, with small height to base diameter ratio, the buoyancy-driven convection can be neglected. Surface forces will play a main role in driving interfacial instability. For binary drops, the excessive free-energy of surface.

$$
F_{E}=(\Delta \sigma) 2 X_{a} X_{b} A /(2 R T)
$$

would occur due to the mixture. Here, $\Delta s$ denotes the surface tension difference of the pure liquid components in the binary liquids; $\mathrm{X}_{\mathrm{a}}$ and $\mathrm{X}_{\mathrm{b}}$, the mole fractions of components $\mathrm{a}$ and $\mathrm{b}$, respectively; and $A$, the partial mole surface area which equals $V_{i}^{2 / 3} N_{0}^{1 / 3} V_{i}$ stands for the mole volume of the pure liquid of component $i$ and $N_{0}$ is the Avogadro number. The excessive free energy of surface in binary liquids will activate the surface phase, leading to the interfacial instability. Hence, the dimensionless parameter

$$
\mathrm{N}_{\mathrm{E}}=\mathrm{F}_{\mathrm{E}} / \sigma_{\mathrm{m}}
$$

could be an important parameter in analyzing interfacial instability for binary drops. The Crispation number

$$
\mathrm{N}_{\mathrm{CR}}=\mu_{\mathrm{m}} \alpha_{\mathrm{m}} /\left(\sigma_{\mathrm{m}} \mathrm{d}_{\mathrm{e}}\right)
$$

is another driving factor for interfacial stability. Here, $\mu_{m}, \alpha_{m}$ and $\sigma_{m}$ denote the dynamic viscosity, thermal diffusivity and surface tension of the binary liquid, respectively. $d_{e}$ is the equivalent diameter of a drop on the test plate at zero time. Because of every present small, random fluctuations in the evaporation rate along the surface, the drop tends to change its surface shape. Large viscosities and lower values of surface tension result in a more difficult recovery of the differential deformation. Hence, $\mathrm{N}_{\mathrm{CR}}$ is another parameter controlling the interfacial flow pattern. The Marangoni effect is generally regarded as a dominant factor driving instability in thin liquid layers and tiny drops. However, it is negligible relative to the effect of excessive free energy of surface in the case of tiny binary drops. The other dimensionless quantities such as the Prandtl, Jakob, Reynolds, Weber and Froude numbers are unimportant [11]. The Crispation number and the dimensionless excessive free energy of surface are calculated for the binary drops tested. They are superimposed on the interfacial flow map $[13,14]$, shown in Fig. 4. The morphology shown in Fig. 3(c) confirms that the prediction that a $50 \%$ dimethyl sulfoxide/50\% oleic acid drop exhibits an unstable type interfacial structure.

\section{1-b. Center cooling case}

The center cooling method produces a temperature field in the drop, low at the summit, increasing toward the base. Hence, the surface temperature gradient induces a thermocapillary force acting from the base toward the drop summit. Low cooling is a rate which produces 


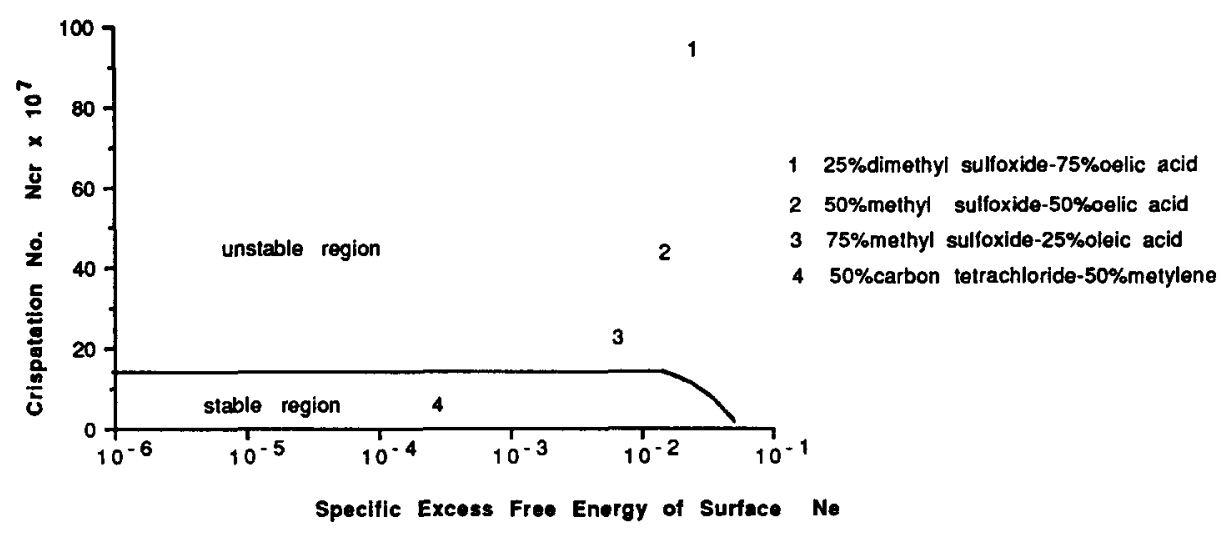

FIG 4

Interfacial flow map of binary liquid drops, $\mathrm{N}_{\mathrm{CR}}$ versus $\mathrm{NE}_{\mathrm{E}}$

component separation without solidification, while high is the opposite, i.e. solidification without component separation. The medium cooling is in between the two, i.e. solidification as well as component separation. Figures 5 and 6 show the morphologies of a $50 \%$ dimethyl sulfoxide/50\% oleic acid drop resulting from low and medium/high heating rates, respectively. A comparison of these figures with Fig. 3 reveals that center cooling, irrespective of cooling intensity, results in the suppression of interfacial structure, from an unstable type (Fig. 3) to a stable one (Fig. 5 and 6). This suppression of surface rippling is due to the action of a strong thermocapillary fore which is induced by a large temperature gradient along the drop surface. The morphology of the dimethyl sulfoxide/oleic acid drop is the same irrespective of mixture compositions.

1-c. Bulk cooling case

Figure 7(a) shows a shadowgraph of a 50\% carbon tetrachloride/50\% methylene chloride drop under bulk cooling. The surface morphology indicates that this binary drop has a stable type interfacial structure which is similar to the one without cooling [18]. Another example is Fig. 8(b), a shadowgraph of a $25 \%$ dimethyl sulfoxide $75 \%$ oleic acid drop under bulk cooling. The binary drop which is characterized by an unstable type interfacial structure under no cooling condition remains unchanged in the presence of bulk cooling. This is because the surface temperature created by this cooling method decreases from the drop summit to the drop base, opposite to that generated by the center cooling method. The resulting temperature gradient induces a thermocapillary force acting from the summit to spread the drop over the test plate, thus exerting little effect on the suppression of interfacial disturbances. 


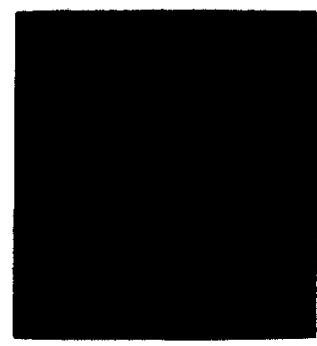

(a)



(d)

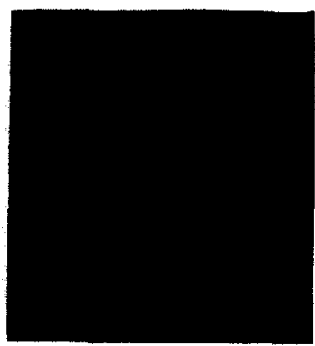

(b)



(e)

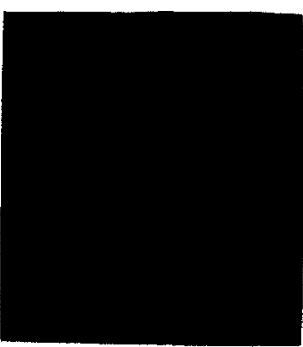

(c)

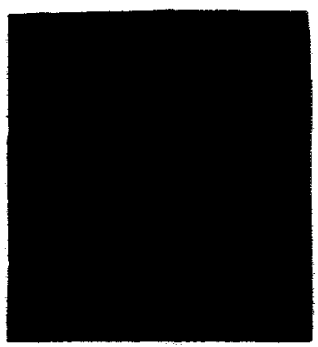

(f)

\section{FIG 5}

Morphology and internal flow pattern in a 50\% dimethyl sulfoxide-50\% oleic acid drop with low center-cooling,



(a)

(b)

(c)

FIG 6

Patterns of separated liquid components: (a) oleic acid evenly distributed in the inner region of dimethyl sulfoxide without solidification, (b) solidification of dimethyl sulfoxide propagated from drop center to edge, (c) unsolidified oleic acid distributed in dimethyl sulfoxide solid and p-xylene crystal 


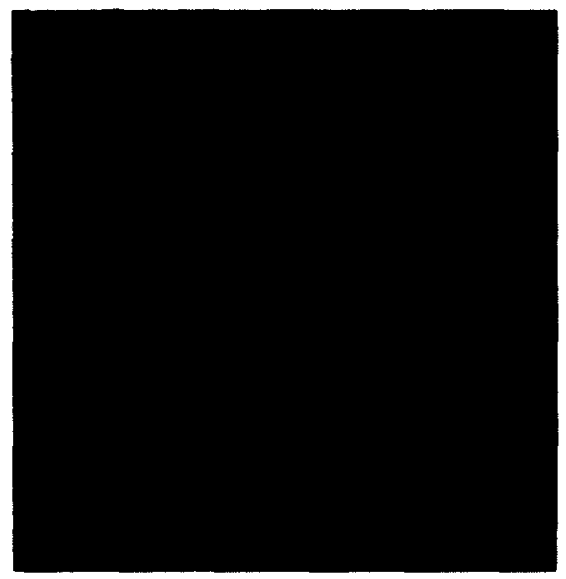

(a)

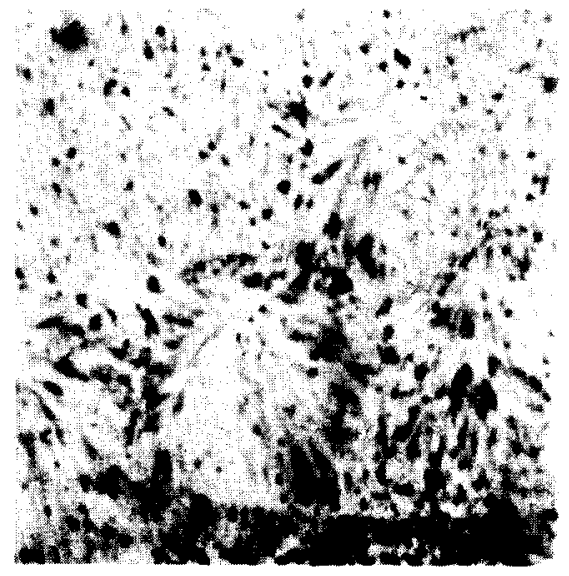

(c)

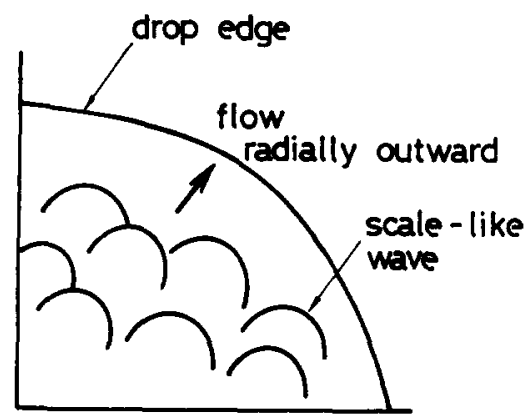

(b)

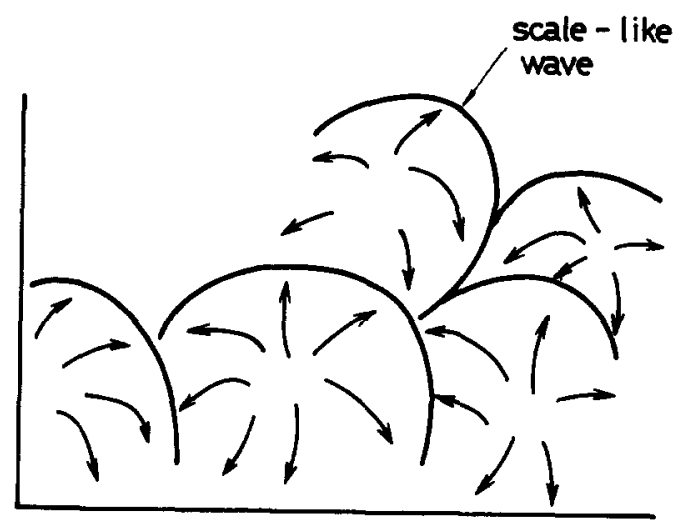

(d)

FIG 7

Morphology and internal flow pattern of a $50 \%$ tetrachloride-50\% methylene chloride drop, (a) shadowgraphic picture, (b) schematic of scale-like waves, (c) flow pattern inside the scale-like waves (d) schematic of (c) 


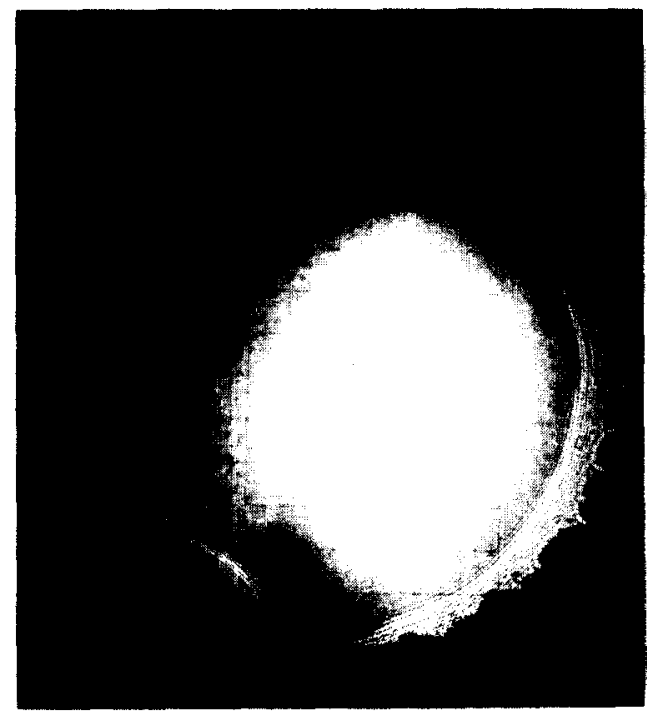

(a)

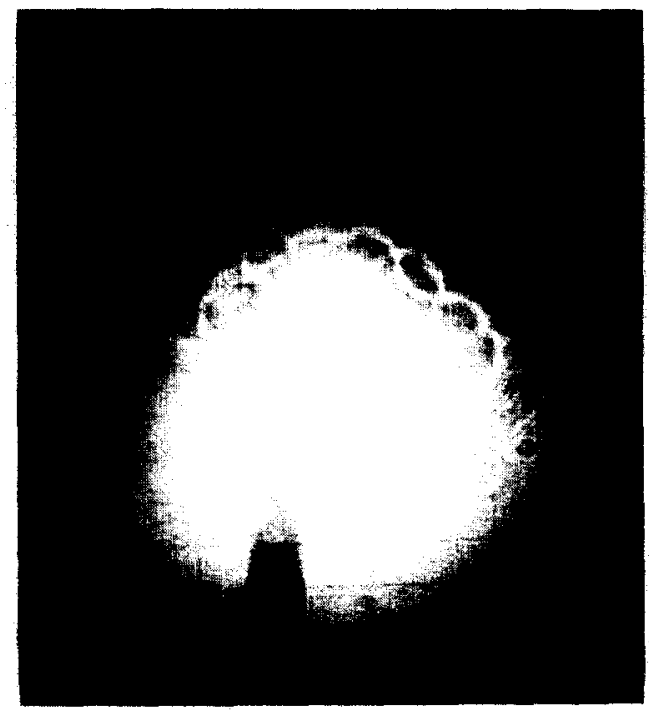

(b)

FIG 8

Morphology and internal flow pattern in a $25 \%$ dimethyl sulfoxide- $75 \%$ oleic acid drop with low bulk cooling in (a) intermediate stage and (b) final stage

\section{Intemal Convection}

No appreciable flow is detected inside an evaporating binary or trinary drops in the absence of cooling.

\section{2-a. Low center cooling}

Figure 5 and 9(a) illustrate the internal flow patterns induced by component separation under low center cooling. No solidification occurs. The sequence (a) to (f) in Fig. 5 ranges from the instant of drop placement on the test plate in (a) to after 30 minutes in (f). Notice the shadow of the conducting wire. The shadowgraph (a) shows a smooth circle which is identical with that in Fig. 3(a). It implies a stable type evaporation. About two minutes after the placement, a circular ring is formed in the middle of the drop shadow, Fig. 5(b), with many slender cells lining up in the radial direction outside the ring. Subsequently, in Fig. 5(c), a second ring is formed inside the first one which deforms and shrinks. Meanwhile, the slender cells grow in length in the radial direction and circular cells begin to form near the outer ring. Figure 5(d) shows the outward migration of the circular cells, disappearance of the slender cells, and deformation of the two concentric rings into the form of a light bulb. Figure 5(b) through (d) mark the intermediate or transition stage. In the final stage, Fig. 5(e), both the circular cells and the inner ring disappear, 

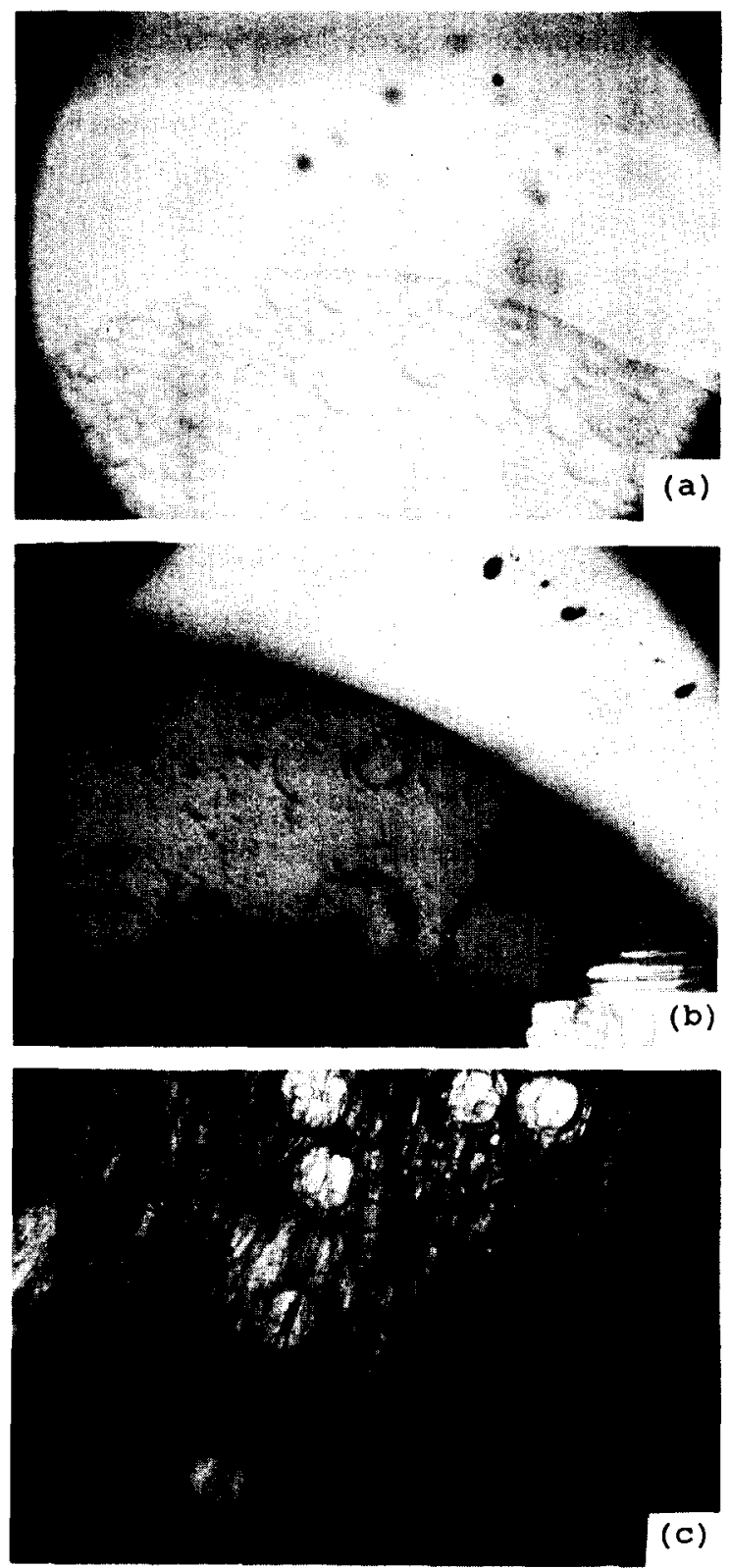

FIG 9

Patterns of separated components: (a) and (b) in $50 \%$ dimethyl sulfoxide $/ 50 \%$ oleic acid drops under center cooling without and with solidification, respectively and (c) in a $25 \%$ oleic acid/25\% dimethyl sulfoxide $/ 50 \%$ p-xylene drop under center cooling 
the slender cells reappear, and the outer ring grows. The drop consists of two regions, being divided by the ring. A placid domain prevails in the inner region and the radial cells distribute in the outer region. Figure 5(f) illustrates the internal flow structure after 30 minutes.

The microscope-video system was employed to investigate the nature of circular cells seen in Fig. 5 . Figure 9(a) shows an enlarged view of a small fraction of Fig. 5(a). The smooth region consists of dimethyl sulfoxide, while the rugged region is a binary mixture in which oleic acid is separated from dimethyl sulfoxide forming individual aggregations due to the action of interfacial surface tension. The aggregations in solid phase obtained by an increased cooling rate are compared with that grown from pure liquids, thus identifying the composition to be oleic acid. Figure 9(b) shows a solidification of dimethyl sulfoxide that has propagated from the drop enter to the edge. It has enclosed some oleic acid aggregates that remain in the liquid phase. A similar picture is shown in Fig. 9(c) for a trinary component drop consisting of $25 \%$ oleic acid, $25 \%$ dimethyle sulfoxide, and $50 \% \mathrm{p}$-xylene. One observes the aggregations of oleic acid in the liquid phase scattering inside the dimethyl sulfoxide solid and p-xylene crystals.

The circular cells in Fig. 5 are the shadow of oleic acid aggregations, while the slender cells represent the intermediate phase of a component separation process. The separation can significantly affect the structure of the solidified phase and crystal. In a binary mixture, the separation may be induced by either Soret diffusion or Gibbs energy. Soret diffusion is a solute flux induced by a temperature gradient. It is unimportant since the center and bulk cooling methods, which generate opposite temperature gradients, produce similar internal flow pattern. The effects of Gibbs energy depend on temperature and composition which induce both thermal and diffuso capillary forces for component separation. In addition, the surface curvature of a binary sessile drop plays an important role in component separation which does not occur in a shallow cavity containing the same binary liquid mixture.

\section{2-b. Medium center cooling}

As the rate of center cooling is increased, a second type of internal flow occurs in the initial stage of the cooling: Marangoni convection induced by surface tension. The flow pattem persists until the onset of component separation: The radially outwand migration of slender cells destroys the Marangoni flow. It is followed by solidification at the base center propagating radially outward. Figure 6(a) depicts internal solidification in the presence of both slender and circular cells. Notice the existence of circular cells surrounding the solid phase (dark color) at the base center. Figure 6(a) corresponds to Fig. 5(c), in the intermediate stage. The solidification propagates radially outward, Fig. $6(\mathrm{~b})$. Meanwhile, the growth of component separation ceases during the course of solidification. 


\section{2-c. High center cooling}

At a high rate of center cooling, component separation ceases to occur. Figure 6(c) shows the drop to consist of the solid phase at the drop center surrounded by the liquid phase. Fine aluminium powders are mixed in multicomponent drops as a tracer. Marangoni convection induced by surface temperature gradients is observed with the aid of the microscope-video system. It forms a doughnut-shaped vortex which is identical with the observed in a pure liquid drop with internal solidification [15-17]. The Marangoni flow velocity is determined by measuring the distance that an arbitrarily selected tracer particle has traveled in a specified time. Figure 10 is a plot of the Marangoni flow velocity versus the composition for dimethyl sulfoxide/oleic acid mixtures. The uncertainty limit is indicated in the plot. The Marangoni flow velocity is retarded with an increase in the Prandtl number or the composition of oleic acid.

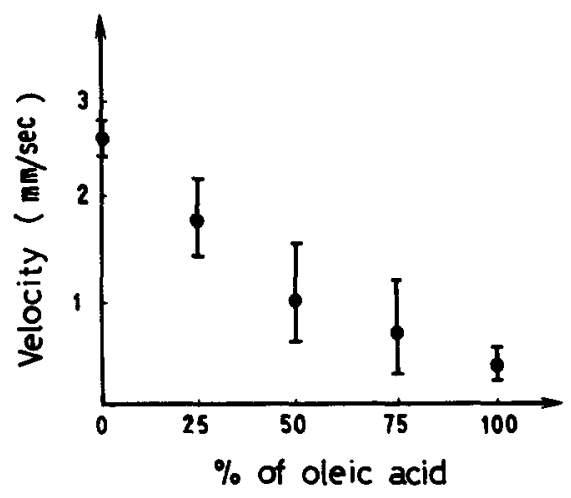

FIG 10

Marangoni flow velocity in dimethyl sulfoxide-oleic acid sessile drops with internal solidification

\section{2-d. Bulk cooling}

The internal flow structure in a 50\% carbon tetrachloride/50\% methylene chloride under high bulk cooling is shown in Fig. 7(a). An observation via the microscope-video system indicates rows of "scales" migrating radially outward. Figure $7(\mathrm{~b})$ is a sketch of "scales" in Fig. 7(a). A close up of a small part of Fig. 7(a) is shown in Fig. 7(c), with its schematic in Fig. 7(d), No component separation is found in this binary drop undergoing a very rapid evaporation. Each "scale" resembles a Benard cell in which the fluid moves upward at the center and down along the cell edge. The flow is much more rigorous than that in pure liquid drops, indicating a significant contribution of diffuso capillary forces. 
Figure 8 depicts the internal flow patterns in a $25 \%$ dimethyl sulfoxide $/ 75 \%$ oleic acid drop under low bulk cooling. The flow behavior is similar to that of the same drop under low center cooling but less slender cells and a larger inner region with enriched oleic aid. No obvious Marangoni convection is observed.

In short, internal convection in binary drops under bulk cooling is similar to that under center cooling.

\section{Conclusions}

A shadowgraphic-schlieren device and a microscope-video system have been employed to determine the mechanics of transport phenomena in multi-component drops under cooling. The shadowgraphic images provide a comprehensive global information of the interfacial structure and internal flow patterns. The microscope-video system supplies the local flow patterns of both Marangoni convection and separation induced flow.

It is disclosed that

(i) In the center cooling method, an increasing surface temperature from the drop summit to the base generates a thermocapillary force acting which suppresses interfacial disturbances.

(ii) In the case of bulk cooling, the opposite temperature field induces a thermocapillary force acting from the drop base toward the summit, exerting little effect on the suppression of interfacial perturbations.

(iii) A low center cooling results in component separation but no solidification and Marangoni convection.

(iv) When the cooling intensity is raised, Marangoni convection appears in the initial stage following the initiation of cooling. The flow is destroyed when slender cells sweep radially outward. It is followed by the radial outward growth of solidified phase.

(v) At a high center cooling rate, component separation fails to occur and Marangoni flow prevails until the arrival of solidification. An increase in the Prandtl number results in a retardation of Marangoni flow velocity.

(vi) Internal convection characteristics in items (iii), (iv) and (v) under center cooling apply to the corresponding cases under bulk cooling. 


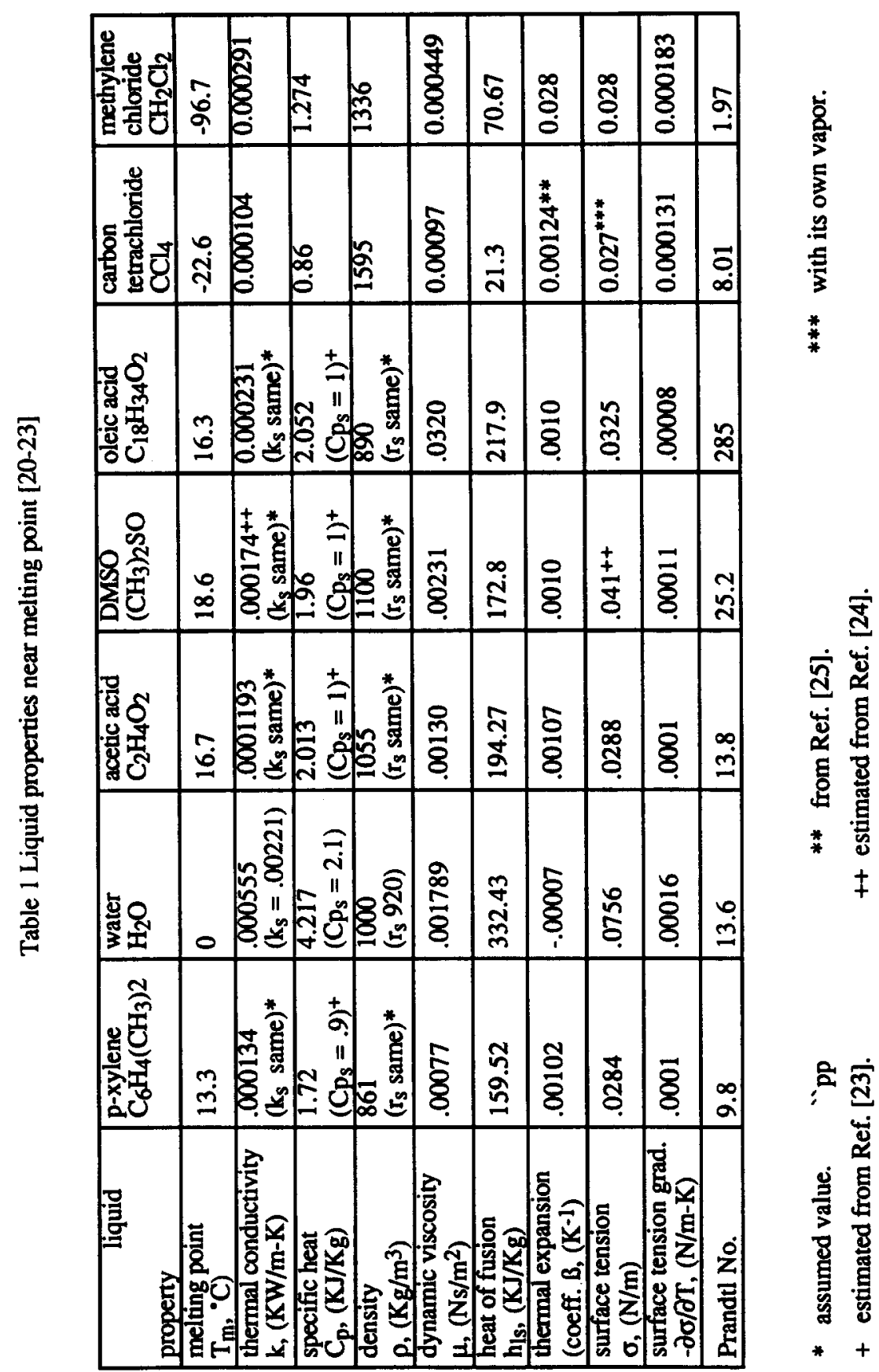




\section{References}

[1] G. Muller, "Convection in Melts and Crystal Growth," Convective Transport and Instability Phenomena, edited by J. Zierep and J. Oertel, Jr., G. Braun, Karlsruhe, . 441 (1982).

[2] C. F. Chen and J. S. Turner, "Crystallization in a Double-Diffusive System," J. Geophs. Res., 85, 2573 (1980).

[3] C. J. Ho and R. Viskanta, "Experimental Study of Solidification in an Open Rectangular Cavity," J. Heat Transfer, 105, pp. 671 (1983).

[4] S. Kou and D. K. Sun, "Flow Field and Weld Penetration in Stationary Arc Welds," Metallurgical Transaction A., 16, . 203 (1985).

[5] S. Ostrach, "Fluid Mechanics in Crystal Growth-the 1982 Freeman Scholar Lecture," J. Fluid Eng., 105, 5 (1983).

[6] M. Epstein and F. B. Cheung, "Complex Freezing-Melting Interfaces in Fluid Flow," Ann. Rew. Fluid Mech., 15, 293 (1983).

[7] M. E. Glicksman, S. R. Coriell and G. B. McFadden, "Interaction of Flows with the Crystal-Melt Interface," Ann. Rew. Fluid Mech., 18, 307 (1986).

[8] Wen-Jei Yang and Y.-J. Su, "Advances in Thermocapillary-Driven Transport Phenomena in Liquids with Solidification," Proceedings of the Second International Symposium on Heat Transfer, 2, 1 (1988).

[9] J. L. Catillo and M. G. Velarde, "Buoyancy-thermocapillary Instability: The Role of Interfacial Deformation in One- and Two-Component Fluid Layers Heated from Below or Above," J. Fluid Mech. 125, 463 (1982).

[10] J. L. Castillo and M. G. Velarde, "Microgravity and Thermo-Convective Stability of a Binary Liquid Layer Open to the Ambient Air," J. Non-Equilibrium Thermodyn., 5, 111 (1980).

[11] N. Zhang and Wen-Jei Yang, "Natural Convection in Evaporating Minute Drops," J. Heat Transfer, 104, 656 (1982).

[12] Wen-Jei Yang and J. C. Duh, "Thermocapillary Flow with Phase Change," proceedings of the Korea-USA Heat Transfer Seminar in Thermal Engineering and High Technology Systems, Oct. 16-22, Seoul, Korea (1986).

[13] N. Zhang, Y. Xu, and Wen-Jei Yang, "Thermal Stability in Binary Droplet Evaporation on a Flat Plate by Real-Time Holographic Interferometry," Heat Transfer 1986-San Francisco, 2, Hemisphere, Washington, D.C., pp. 525-530 (1986).

[14] N. Zhang, B. X. Wang and Y. Xu, "Thermal Instability of Evaporating Drops on a Flat Plate and Its Effects on Evaporation Rate," Int. J. Heat Mass Transfer, 30, 469 (1987).

[15] Y.-J. Su, Wen-Jei Yang, and G. Kawashima, "Natural Covection in Evaporating Sessile Drops with Solidification," Int. J. Heat Mass Transfer, 31, 375 (1988).

[16] Y.-J. Su, "Thermocapillary Convection in Evaporating Sessile Drops with Internal Solidification," Ph.D. Dissertation, Dept. of Mech. Eng. and Applied Mechanics, University of Michigan, Ann Arbor, 1988. 
[17] Y.-J. Su and Wen-Jei Yang, "Thermocapillary Convection in Evaporating Sessile Drops with Internal Solidification," to be presented at the 9th Int. Heat Transfer Conf., Jerusalem, August 19-24 (1990).

[18] N. Zhang and Y. Zu, "Visualization of Evaporative Convective in Binary Drop by Laser shadowgraphy and Holographic Interferometry," Flow Visualization $I V$, edited by Claude Veret), Hemisphere, Washington D.C., pp. 685-690 (1987).

[19] Y.-J. Su and Wen-Jei Yang, "Visualization of Marangoni Effects in Evaporating Sessile Drops with/without Internal Solidification", Flow Visualization V (edited by R. Reznicek) Hemisphere, Washington D.C. (1990).

[20] R. C. Weast, CRC Handbook of Chemistry and Physics, 66th ed., CRC Press Inc., Boca Raton, Florid (1985).

[21] C. L. Yaws, Physical Properties, McGraw-Hill, New York (1977).

[22] K. Raznjevic, Handbook of Thermodynamic Tables and Charts, Hemisphere Publishing Corp., Washington D.C. (1976).

[23] K. Othmer, Ed., Encyclopedia of Chemical Technology Vol. 22, 3rd ed., John Wiley \& Sons (1983).

[24] W. J. Lyman, W. F. Reehl, and D. H. Rosenblatt, Handbook of Chemical Property Estimation Methods, McGraw-Hill, New York (1982).

[25] J. A. Dean,, "Lange's Handbook of Chemistry", 13th edition, McGraw-Hill, New York (1972). 